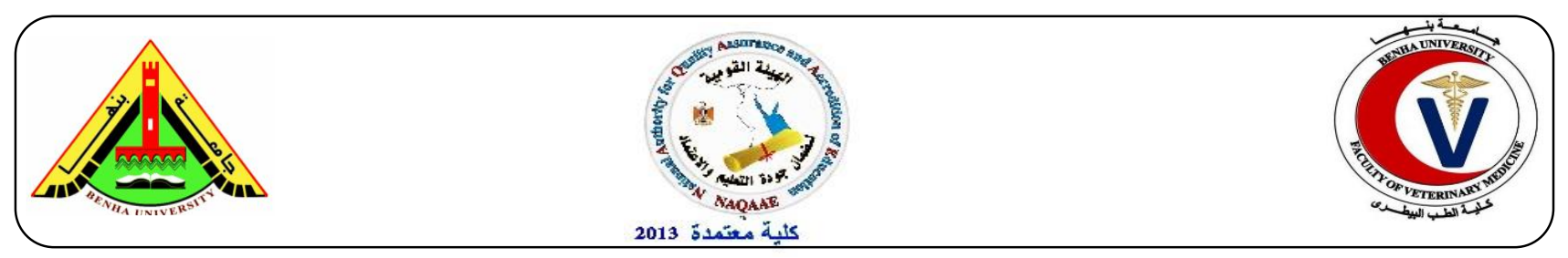

\title{
Avocado Oil Attenuates Diethylnitrosamine Induced-Hepatic Carcinogenesis
}

\author{
Abozaid O.A.R. ${ }^{1}$, Aziza S.A.H. ${ }^{1}$, Moawed F. S.M², Kasseb S.G. ${ }^{1}$ \\ ${ }^{1}$ Department of Biochemistry, Faculty of Veterinary Medicine, Benha University, Egypt. \\ ${ }^{2}$ Health radiation research, National Center for Radiation Research and Technology, Atomic \\ Energy Authority, Cairo, Egypt.
}

\section{A B S T R A C T}

This study aimed to investigate the efficacy of avocado oil against diethylnitrosamine (DEN) induced hepatocarcinogenesis in rats. Rats were divided into 5 groups. Group (1) was negative control. Groups (2) and (4) were orally administrated diethylnitrosamine for induction of hepatocellular carcinoma then group (2) was left untreated; group 4 was treated orally with avocado oil before DEN administration. Group (3) was orally treated with avocado oil only. Serum alphafetoprotein (AFP) was assayed using ELISA technique. The untreated cancer group showed significant elevation in the liver function (ALT, AST, ALP, total bilirubin and direct bilirubin) and tumor marker AFP. Histopathological investigation of liver tissue sections in cancer group revealed dysplasia. In contrast, the treated groups showed significant depletion in the liver function and AFP and significant decrease in the liver function (ALT, AST, ALP, total bilirubin and direct bilirubin). Interestingly, treatment with avocado oil showed marked improvement in the histological feature of liver tissue. It is concluded that this study indicated the promising therapeutic potential of avocado oil against DEN-induced hepatocarcinogenesis. Further studies are required to evaluate the possible mechanism at the molecular level.

Key words: Diethylnitrosamine, hepatocarcinogenesis, Avocado oil, liver function, tumor marker (http://www.bvmj.bu.edu.eg)

(BVMJ-35(2): 344-353, 2018)

\section{INTRODUCTION}

Hapatocellular carcinoma (HCC) is the most frequent primary malignancy of the liver. It accounts for about $90 \%$ of all liver cancer and it represents more than $4 \%$ of all cancer cases worldwide and is the fourth most common cause of cancer mortality. Most major well known risk factors of hepatocellular carcinoma includes hepatitis viral infection (HBV and $\mathrm{HCV}$ ), food additives, alcohol, fungal toxins (aflatoxins), toxic industrial chemicals, and air and water pollutants. In many patients, HCC is 
asymptomatic and when symptoms occur they are usually related to those of chronic liver disease such as yellowing of the skin and eyes, pain in the right upper abdominal side, swelling of the abdomen, weakness, weight loss and finally fever (Raphael SW et al., 2012).

Diethylnitrosamine (DEN, NNitrosodiethylamine) a potent hepatocarcinogen, is known to cause perturbations in the nuclear enzymes involved in DNA repair/replication (Bhosale et al., 2002). N-nitroso compounds are considered to be a tragedic health hazards to man, and these compounds were present in tobacco products, cheddar cheese, cured and fried meals, occupational settings, cosmetics, agricultural chemicals, and pharmaceutical agents. It has been suggested that DEN, after its metabolic activation produces the promutagenic adducts, O6 -ethyl deoxy guanosine and 04 and O6 -ethyl deoxy thymidine in liver that may cause carcinogenic effects. It is also reported that, the oxidative stress plays a causative role during carcinogenesis (Jayakumar et al., 2012). Accumulating evidence has demonstrated that overproduction of the formation of reactive oxygen species (ROS) during the metabolic biotransformation of DEN plays a key role in the etiology of hepatocarcinoma. ROS could result in oxidative damage of DNA, lipid and protein damage, as well as a change in the intracellular signaling pathways, which facilitate the formation of hepatocellular carcinoma (HCC) (Shahin R, et al., 2018).

Avocado is the most commonly sold fruits in the world and its nutritional content depends on fruit variety and the season of the year. Avocado contains one to two times more protein than any other fruits. It has higher content of iron, phosphorous, manganese and potassium, while it is low in sodium. Avocado is loaded with nutrients such as vitamins $\mathrm{E}, \mathrm{C}$, thiamin, riboflavin, nicotinic acid and folate as well as -carotene. It is an excellent source of monounsaturated fat and is a good source of the essential linoleic acid. It contains several structural polysaccharides, including cellulose and lignin (insoluble fiber) and hemicelluloses and pectin (soluble fiber). Even though avocado has been used for centuries as a herbal medicine and its anticancer effects on various cancers are studied as a hepatoprotective (Mahmoed and Rezq, 2013), antiosteoarthritis (Christiansen et al., 2015) and chemo-protective (Paul et al., 2011). Its effect on hepatocarcinogenesis is not yet documented. In this investigation, we evaluated efficacy of avocado oil on DENinduced hepatocarcinogenesis in rats.

\section{MATERIALS AND METHODS}

2.1. Chemicals

DEN and carvacrol were purchased from Sigma Chemicals Co. (St. Louis, MO, USA). All other chemicals used were of analytical grade. 
2.2. Avocado Oil Extraction: The oil was obtained from Hass avocado purchased from a local market in Cairo, Egypt. When edible maturity had been reached, the avocados were washed and peeled and the seed removed. Subsequently, the pulp was homogenized by adding tert-butylhydroquinone (TBHQ) at $0.1 \% \quad(\mathrm{w} / \mathrm{w})$ then Centrifugation and separation oil from other precipitate.

\subsection{Animals}

Male Wistar rats (weighing 110-120 g) were obtained from the Nile Pharmaceutical Co., Cairo, Egypt. They were housed at the animal facility at the National Center for Radiation Research and Technology. Upon arrival, the animals were allowed to acclimatize for one week before starting the experiment. The animals were kept under standard laboratory conditions of light/dark cycle $(12 / 12 \mathrm{~h})$, a temperature of $25 \pm 2{ }^{\circ} \mathrm{C}$ and humidity of 60 $\pm 5 \%$. The rats were housed in cages with free access to food and drinking water ad libitum. They were provided with a nutritionally adequate standard laboratory (pellet) diet. The study was conducted in accordance with international guidelines for animal experiments and approved by the Ethical Committee at the National Center for Radiation Research and Technology (NCRRT), Atomic Energy Authority, Cairo, Egypt.

\subsection{Experimental Design}

Rats were randomly divided into four main equal groups, 6 animals each, placed in individual cages and classified as follow:
Group (1): Normal control group

Rats received normal saline, served as untreated control for all experimental groups. Group (2): DEN group

Animals were orally administered DEN (dissolved in $0.9 \%$ normal saline), in a dose of $20 \mathrm{mg} / \mathrm{kg}$ b.w. five times a week for six weeks according to the modified method of (Darwish and El-Boghdady, 2011).

Group (3): Avocado Oil normal treated group Rats were administrated with Avocado Oil ( $1 \mathrm{~mL} / 250 \mathrm{~g}$ b.wt/day, orally) daily for 6 weeks.

Group (4): DEN + Avocado Oil protected group

Rats were pretreated with Avocado Oil (1 mL/250 g b.wt/day, orally) daily for 6 weeks. Then Rats injected with DEN (20 $\mathrm{mg} / \mathrm{Kg}$ b.wt, orally) as in group 2 and continuously treated with avocado oil (end of experiment).

At the end of the experiment, blood samples were collected after overnight $12 \mathrm{hr}$ fasting from the retro-orbital venous plexus located at the medial canthus of the eye using heparinized capillary tubes, in dry, clean screw-copped tubes then coagulated at room temperature for 30 minutes and separated by centrifugation at 3000 r.p.m. around 15 minutes. The clean, clear serum was separated by automatic micropipettes and received in dry sterile Eppendorf's tubes and processed directly for determination of the biochemical parameters.

2.5. Determination of liver function in serum 
Serum alanine aminotransferase (ALT), aspartate aminotransferase (AST) (Bergmeyer et al., 1986), alkaline phosphatase (ALP) (Tietz et al., 1983), total protein (Burtis et al., 2012), total and direct bilirubin were determined using commercially available kits (Spinreact, Santa Coloma, Spain) (Kaplan A et al., 1984).

2.6. Determination of Alpha-fetoprotein (AFP) concentration in serum

Serum alpha-fetoprotein was estimated by enzyme linked immunosorbent assay (ELISA) using a rat alpha- fetoprotein (AFP) ELISA kit purchased from Glory Science Co., Ltd (USA) (Cattini et al., 1993).

\subsubsection{Histopathological Study}

Specimen from kidney of all examined groups were washed, dehydrated in ascending grades of ethyl alcohol, cleared in xylene and embedded in paraffin wax. Sections of 5-6 $\mu \mathrm{m}$ in thickness were cut out, deparaffinized and stained with Hematoxylin and Eosin $(\mathrm{H}$ \& E) for examination under the light microscope (Banchroft et al., 1996)

\subsubsection{Statistical Analyses.}

The SPSS (version 20) was used in data analyses. Data were analyzed with one-way analysis of variance (ANOVA) followed by a post hoc test (LSD) for multiple comparisons. The data were expressed as mean \pm standard error $(\mathrm{SE})$. $\mathrm{P}$ values $<0.05$ were considered statistically significant.

\section{RESULTS}

\subsection{Biochemical studies}

To investigate the effect of avocado oil on DEN-induced liver carcinogenesis, activities of ALT, AST and ALP were measured in the serum of both avocado oil-treated and untreated animals. As shown in Table 2, DEN administration resulted in severe liver injury as manifested by significant increase in serum activities of ALT, AST, and ALP compared to control, which was attenuated by avocado oil administration compared to DEN group.

The levels of total protein, total bilirubin and direct bilirubin were measured in the serum of both avocado oil-treated and untreated animals. As shown in Table 2, DEN administration resulted in severe liver injury as manifested by significant decrease in serum level of total protein with increase in total bilirubin and direct bilirubin levels compared to control, which was ameliorated by avocado oil administration compared to DEN group.

As shown in Fig. 1, serum level of AFP was significantly elevated in DENtreated group compared to normal rats. While, the treatment with avocado oil of DEN treated rats modulated the level of AFP compared to DEN-treated animals.

\subsection{Histopathological observation}

Histopathological examination of liver sections of control rats showed normal hepatic architecture (Fig.2A). The liver portal area of rats administrated with DEN 
showed inflammatory cells infiltration and congestion in the portal vein in association with degeneration and dysplasia in the hepatocytes all over the hepatic parenchyma (Fig.2B). The hepatic parenchyma of rats received avocado oil only showed normal architecture (Fig. 2C). Pretreatment of rats with avocado oil with injection of rats with DEN revealed mild reduction in the neoplastic changes that were observed in DEN injected group as large number of abnormal hepatocytes was observed containing irregular clear vacuoles. In addition, individual dysplastic changes was found (anisonucleosis, irregular chromatin pattern and prominent nucleoli was noted) (Fig.2D).

Table 1: Effect of avocado oil and/or DEN on ALT, AST and ALP in normal rats

\begin{tabular}{cccc}
\hline & ALT & AST & ALP \\
& $(\mathrm{U} / \mathrm{L})$ & $(\mathrm{U} / \mathrm{L})$ & $(\mathrm{U} / \mathrm{L})$ \\
NORMAL & $11.5 \pm 1.5$ & $15.0 \pm 1.0$ & $123.0 \pm 2.0$ \\
DEN & $55.5 \pm 6.5^{\mathrm{a}}$ & $63.5 \pm 8.5^{\mathrm{a}}$ & $262.5 \pm 45.5^{\mathrm{a}}$ \\
AVOCADO & $13.0 \pm 1.0^{\mathrm{b}}$ & $11.0 \pm 1.0^{\mathrm{b}}$ & $121.0 \pm 2.0^{\mathrm{b}}$ \\
$\begin{array}{c}\text { AVOCADO+DEN } \\
\text { (prevented) }\end{array}$ & $25.0 \pm 4.0^{\mathrm{b}}$ & $21.5 \pm 1.5^{\mathrm{b}}$ & $170.5 \pm 2.5^{\mathrm{b}}$ \\
\hline
\end{tabular}

Data are presented as (Mean \pm S.D). Mean values with different superscript letters in the same column are significantly different at $(\mathrm{P} \leq 0.05)$. a: Significance against normal control group $(\mathrm{P} \leq 0.05), \quad \mathrm{b}$ : Significance against $\mathrm{DEN}$ group $(\mathrm{P} \leq 0.05)$.

Table 2: Effect of avocado oil and/or DEN on total protein, total bilirubin and direct bilirubin in normal rats

\begin{tabular}{cccc}
\hline & $\begin{array}{c}\text { Total Protein } \\
(\mathrm{gm} / \mathrm{dl})\end{array}$ & $\begin{array}{c}\text { Total bilirubin } \\
(\mathrm{mg} / \mathrm{dl})\end{array}$ & $\begin{array}{c}\text { Direct bilirubin } \\
(\mathrm{mg} / \mathrm{dl})\end{array}$ \\
NORMAL & $5.35 \pm 0.25$ & $0.88 \pm 0.02$ & $0.25 \pm 0.02$ \\
DEN & $4.16 \pm 0.15^{\mathrm{a}}$ & $1.90 \pm 0.20^{\mathrm{a}}$ & $0.77 \pm 0.02^{\mathrm{a}}$ \\
AVOCADO & $5.65 \pm 0.15^{\mathrm{b}}$ & $0.87 \pm 0.05^{\mathrm{b}}$ & $0.23 \pm 0.01^{\mathrm{b}}$ \\
$\begin{array}{c}\text { AVOCADO+DEN } \\
\text { (prevented) }\end{array}$ & $4.75 \pm 0.15$ & $1.46 \pm 0.15^{\mathrm{ab}}$ & $0.47 \pm 0.05^{\mathrm{ab}}$ \\
\hline
\end{tabular}

Data are presented as (Mean \pm S.D). Mean values with different superscript letters in the same column are significantly different at $(\mathrm{P} \leq 0.05)$. a: Significance against normal control group $(\mathrm{P} \leq 0.05), \quad \mathrm{b}$ : Significance against $\mathrm{DEN}$ group $(\mathrm{P} \leq 0.05)$. 


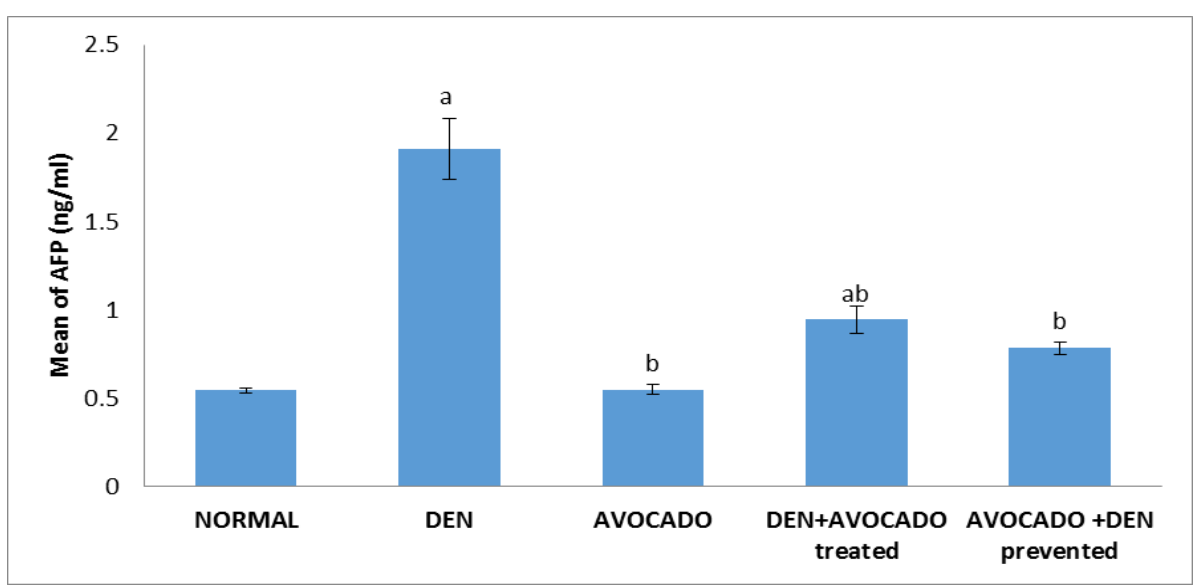

Fig.1: Effect of avocado oil on serum AFP concentration in hepatocarcinogenesis induced in rats. $\mathrm{a}=$ Significance against normal control group $(\mathrm{P} \leq 0.05), \quad \mathrm{b}=$ Significance against DEN group $(\mathrm{P} \leq 0.05)$.

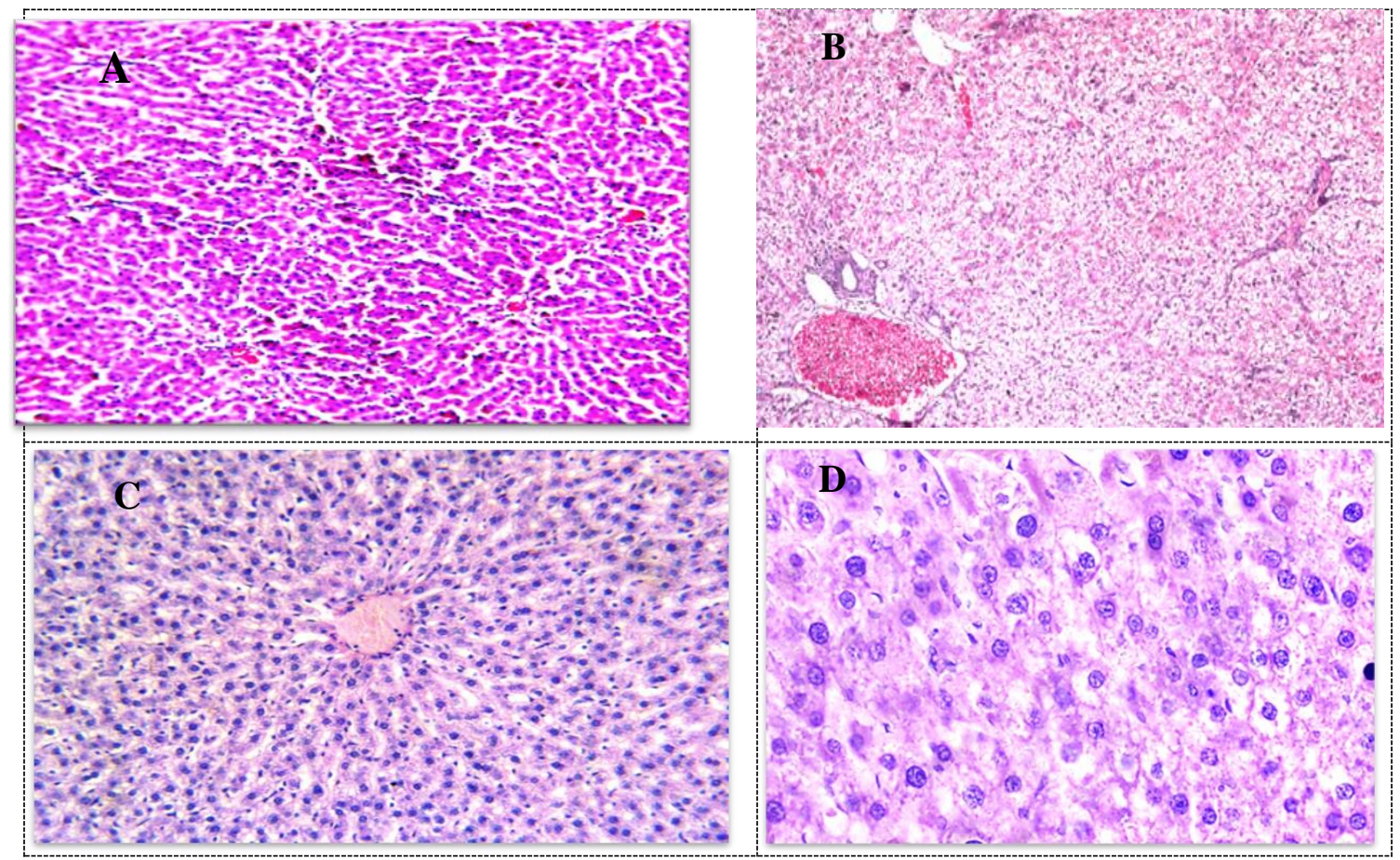

Fig. 2: Light microscopy of liver sections showing: (A) Control group; A normal rat liver tissue), (B) DEN-treated group; liver of rat in DEN group, showing congestion of portal vein with inflammatory cells infiltration in portal area as well as degeneration and dysplasia in the hepatocytes all over the parenchyma (H.E x16). (C) Avocado oil group; A liver of rat receiving avocado showing unremarkable changes (H\&E x200) (D) Avocado oil+ DEN; A liver of rat receiving DEN after avocado showing dysplastic changes (the anisonucleosis, irregular chromatin pattern and prominent nucleoli was noted) (H\&E x400). 


\section{DISCUSSION}

To our knowledge, the current study is considered the first report showing chemopreventive effect of avocado oil on DEN-induced hepatocellular carcinogenesis in rats. In this study, the pretreatment of avocado oil to HCC rats caused a remarkable reduction in the serum markers of liver damage and cancer. The biomarkers implicated by the obtained data presented in this study include increased serum ALT, AST, ALP, total protein, total and direct bilirubin and AFP. Serum total protein decrease. Meanwhile, each individual marker is not specific for liver cancer; the collective changes in all of these parameters appear to correlate with DEN treatment, with liver tumor development, and with the effect of avocado oil on DEN-induced carcinogenesis.

Elevations of activities of ALT and AST can indicate the presence of hepatocellular predominant disorders while elevations of ALP activity may implicate cholestatic predominant diseases (Eissa et al., 2018). Recently, the effects of these three commonly tested serum liver enzymes were evaluated on the long-term risk of HCC. The HCC incidence rates were higher in the elevated groups for all three enzymes. Similar results were obtained in DEN-induced HCC in albino rats that is proved in many studies like (Carvajal-Zarrabal et al., 2014) who reported that serum biochemical analyses were performed to determine hepatic function. ALT, AST and ALP activities showed a significant increase in DEN-treated group.

DEN-treated rats had elevated serum bilirubin level (total and direct) compared with the normal rats. Similar results were observed in many studies such as (Rui et al., 2014) who reported that, DEN administration resulted in serum total bilirubin of HCCinduced albino rats. The hepatocarcinogensis induced by DEN exposure for rats exhibited significant decrease in total protein concentrations when compared with the normal rats group.

In the present study, treatment with avocado oil on DEN-induced hepatocarcinogenesis in rats exhibited a significant decrease in serum ALT, AST, ALP when compared with the DEN group. Similar effects were reported by (Mahmoed and Rezq, 2013) who found reduced liver enzymes AST, ALT and ALP and mortality after avocado oil administration, which was attributed to downregulation of hepatocellular apoptosis in septic mice accordingly; avocado oil improved liver functions and showed antioxidant activity. Also (Zhao et al. 2015) reported that DEN-administered rats in the present work demonstrated high levels of liver enzymes (AST and ALT) and augmented content of total bilirubin and ALP in serum. In addition to Similar effects were reported by (Carvajal-Zarrabal et al., 2014) 
who found reduced liver enzymes after avocado oil administration, which was attributed to avocado oil are recognized as oil with a high percentage of unsaturated fatty acids and a low percentage of saturated (Wong et al., 2010) in rats models, different transcriptomic responses between diets based on different long-chain polyunsaturated fatty acids have been observed. Furthermore, stereochemistry influences differential responses as seen with linoleic acid isomers (Osada et al 2013). This results showed agreement with results reported by (Oyeyemi and Oyeyemi, 2015) who observed that a significant decrease in the activities of ALP, ALT and AST in the liver and plasma of the rats. The three levels also decreased which indicated the hypocholesterolimic effect of avocado oil. Treatment with avocado oil exhibited a significant decrease in serum total, direct bilirubin when compared with the DEN group and that may be due to the antioxidant properties of avocado oil that counteracted the toxic effects of DEN on serum bilirubin (Attia et al., 2010). Treatment with avocado oil in liver cancer induced rats exhibited a significant increase in serum total protein concentration when compared with the DEN group.

AFP level has been widely used clinically as a tumor marker for HCC that was markedly enhanced in DEN-treated rats compared with normal animals Serum AFP level is the golden standard among diagnostic markers for HCC (Chou et al., 2017). In the present study, treatment with avocado oil exhibited a significant decrease in serum AFP when compared with the DEN group and that may be due to the anti-inflammatory properties of avocado oil that regulates the inflammatory response and immune function and the anticancer properties of avocado oil (Lu et al., 2005; Sudhir, 2005).

\section{CONCLUSION}

It is concluded that avocado oil can be considered a promising therapeutic adjuvant against DEN-induced hepatocarcinogenesis. Further studies are required to evaluate the molecular targeting of avocado as chemopreventive agent against hepatocarcinogenesis.

\section{ACKNOWLEDGEMENTS}

We wish to acknowledge the contribution of Dr. Laila Rashed, Prof of Biochemistry, Faculty of Medicine, Cairo University and Dr. Osama Mostafa, lecturer of Pathology, Faculty of Medicine, Al-azhar University, for assistance in setting up histopathological study.

\section{REFERENCES}

Attia, A. M. and Nasr, H. M. (2009). Evaluation of protecrive effect of omega 3 fatty acids and selenium on paraquat intoxicated rats. Slovak J. Anim. Sci; 42 (4): 180 - 187.

Banchroft, J. D., Stevans, A., \& Turnes, D. R. (1996). Theory and Practice of Histological Techniques 4th ed., livingstone. Edinburgh, London, Melbourne, New York, Tokyo.

Bergmeyer, H.U.; Hørder, $\mathrm{M}$ and Rej. R. (1986). International Federation of Clinical Chemistry (IFCC) Scientific 
Committee, Analytical Section: approved recommendation (1985) on IFCC methods for the measurement of catalytic concentration of enzymes. Part 3. IFCC method for alanine aminotransferase (L-alanine: 2oxoglutarate aminotransferase, EC 2.6.1.2). J Clin Chem Clin Biochem.; 24:481-495.

Bhosale P, Motiwale L, Ignle AD, Gadre RV, Rao KVK (2002) Protective effect of Rhodotorula glutinis NCIM3353 on the development of hepatic preneoplastic lesions. Curr Sci 83:303308

Burtis, C.A., Ashwood, E.R. and Bruns, D.E. (2012): Tietz Textbook of Clinical Chemistry and Molecular diagnostics 5th edition.

Carvajal-Zarrabal, O., Nolasco-Hipolito, C., Aguilar-Uscanga, M., Melo Santiesteban, G., Hayward-Jones, P. M., \& Barradas-Dermitz, D. M. (2014). Effect of dietary intake of avocado oil and olive oil on biochemical markers of liver function in sucrose-fed rats. BioMed research international, 2014.

Cattini, R.; Cooksey, M.; Robinson, D.; Brett, G.; Bacarese-Hamilton, T. and Jolley, N. (1993): Measurement of alpha-fetoprotein, carcinoembryonic antigen and prostate-specific antigen in serum and heparinised plasma by enzyme immunoassay of the fully automated serono SR1TM analyzer. Eur. J. Clin. Chem. Clin. Biochem., 31(8): 517-524.

Chou WC, Lee CL, Yang TS, Huang CY, Teng W, Tseng YT 2017. Changes in serum $\alpha$-fetoprotein level predicts treatment response and survival in hepatocellular carcinoma patients and literature review. J Formos Med Assoc. 2017; [Epub ahead of print.
Christiansen BA, Bhatti S, Goudarzi R, Emami S. 2015. Management of osteoarthritis with avocado/soybean unsaponifiables. Cartilage 6: 30-44.

Darwish, H. A., \& EL-BOGHDADY, N. A. (2013). Possible involvement of oxidative stress in diethylnitrosamineinduced hepatocarcinogenesis: chemopreventive effect of curcumin. Journal of Food Biochemistry, 37(3), 353-361.

Eissa, L. A.; Kenawy, H. I.; El-Karef, A.; Elsherbiny, N. M. and El-Mihi, K. A. (2018): Antioxidant and antiinflammatory activities of berberine attenuate hepatic fibrosis induced by thioacetamide injection in rats.Chemico-Biological Interactions.294 (91-100).

Jayakumar S1, Madankumar A, Asokkumar S, Raghunandhakumar S, Gokula dhas K, Kamaraj S, Divya MG, Devaki T. Potential preventive effect of carvacrol against diethylnitrosamine-induced hepatocellularcarcinoma in rats. Mol Cell Biochem. 2012 Jan; 360(1-2):5160.

Kaplan A, 1984. Bilirubin. Clin Chem the C.V. Mosby Co. St Louis. Toronto. Princeton 1984; 1238-1241. 436: 650.

LU, N. Z, Bohn T, Clinton SK, Schwartz SJ 2005. Carotenoid absorption from salad and salsa by humans is enhanced by the addition of avocado or avocado oil. $\mathbf{J}$ Nutr; 135: 431-436 [PMID: 15735074].

Mahmoed M. Y. and Rezq A. A. (2013), Hepatoprotective Effect of Avocado Fruits Against Carbon TetrachlorideInduced Liver Damage in Male Rats World Applied Sciences Journal 21 (10): 1445-1452, 2013

Osada, J. (2013). The use of transcriptomics to unveil the role of nutrients in mammalian liver. ISRN nutrition, 2013. 
Oyeyemi A, Oyeyemi R. 2015. Effect of the aqueous extract of the leaves and seeds of avocado pear (Persea Americana) on some marker enzymes and cholesterol in the albino rat tissues. IOSR J Environ Sci Toxicol Food Technol 9: 15-18.

Paul R, Kulkarni P, Ganesh N. 2011. Avocado fruit (Persea americana Mill) exhibits chemo-protective potentiality against cyclophosphamide induced genotoxicity in human lymphocyte culture. J Exp Ther Oncol 9: 221-230.

Raphael SW, Yangde Z, and YuXiang C. Hepatocellular Carcinoma2012: Focus on Different Aspects of Management ISRN Oncology. Article ID 421673:12.

Rui, W.; Xie, L.; Liu, X.; He, S.; Wu. C.; Zhang, X.; Zhang, L and Yang, Y. (2014). Compound Astragalus and Salvia miltiorrhiza extract suppresses hepatocellular carcinoma progression by inhibiting fibrosis and PAI-1 mRNA transcription. J Ethnopharmacol; 151:198-209.

Shahin, Y. R., Elguindy, N. M., Abdel Bary, A., \& Balbaa, M. (2018). The protective mechanism of Nigella sativa against diethylnitrosamine-induced

epatocellular carcinoma through its antioxidant effect and EGFR/ERK1/2 signaling. Environmental Toxicology.

Sudhir K. 2005. Lipoprotein-associated phospholipase A2, anovel inflammatory biomarker and independent risk predictor for cardiovascular disease. $\mathbf{J}$ Clin Endocrinol Metab 90:3100-3105.

Tietz, N.W.; Burtis, C.A.; Ducan, P.; Ervin, K.; Petitclera, C.J.; Rinker, A.D.; Shney, D. and Zygowicz, E.R. (1983). A reference method for measurement of alkaline phosphatase activity in human serum. Clin. Chem.; 29:751-761.

Wong, M., Requejo-Jackman, C., \& Woolf, A. (2010). What is unrefined, extra virgin cold-pressed avocado oil. J. Am. Oil Chem. Soc, 87, 1099.

Zhao, X., Chen, Q., Li, Y., Tang, H., Liu, W., and Yang, X. 2015. Doxorubicin and curcumin co-delivery by lipid nanoparticles for enhanced treatment of diethylnitrosamine-induced

epatocellular carcinoma in mice. Eur. J. Pharm. Biopharm. 93: 27-36. doi:10.1016/j.ejpb.2015.03.003. 